\title{
Study of Clinical Spectrum and Management of Hydatid Disease- A 7 Years' Prospective Study
}

\author{
Brajesh B Gupta ${ }^{1}$, Pavan Kumar C G ${ }^{2}$ \\ ${ }^{1}$ Professor, ${ }^{2}$ Junior resident, Department of General Surgery, Government Medical College and Hospital, Nagpur, Maharashtra, \\ India
}

Corresponding author: Dr Pavan Kumar C G, Junior Resident, Department of General Surgery, Government Medical College and Hospital Nagpur, Maharashtra, India

DOI: 10.21276/ijcmsr.2018.3.2.16

How to cite this article: Brajesh B Gupta, Pavan Kumar C G. Study of clinical spectrum and management of hydatid disease- a 7 years' prospective study. International Journal of Contemporary Medicine Surgery and Radiology. 2018;3(2):B65-B69.

\section{A B S T R A C T}

Introduction: Hydatid disease is a zoonotic disease caused by the tapeworm of genus Echinococcus species and is one of the oldest known diseases to man. The primary management involves surgical removal. We conducted this prospective cohort study to describe the epidemiology (demography, clinical presentation, imaging characteristics, management and in-hospital course) of Cystic Echinococcosis (CE) in central India.

Material and Methods: The study was approved by institutional ethical committee and was conducted in the department of general surgery at a tertiary care medical college situated in an urban area. A Total of 148 Patients diagnosed with hydatid disease were included in this study on the basis of predefined inclusion criteria. Any patient having any exclusion criteria was excluded from the study. The diagnosis was confirmed on the basis of imaging findings. Patients were treated primarily by surgery (Open or laparoscopic depending upon the location and size). Statistical analysis was done with Minitab version 17.

Results: Out of total 148 patients there were 46 males and 102 females with a M: F ratio of 1:2.21. Majority of the patients $(55.40 \%)$ were operated by endoscopic techniques while open surgeries were done in $44.60 \%$ patients. Amongst the surgeries done for hepatic hydatid cysts laparoscopic and open surgeries were done in $70(70 \%)$ and 30 (30\%) patients respectively with $11 \%$ conversion rate. Out of the patients with lung hydatids $70 \%$ were operated by thoracotomy while $30 \%$ were operated with VATS With $20 \%$ conversion. Splenectomy was done in $85.71 \%$, and cystectomy in $14.29 \%$ of splenic hydatid patients.

Conclusions: Management of the hydatid cyst can safely be done by laparoscopic surgery and VATS with acceptable complication rates.

Keywords: Hydatid Disease, Laparoscopic Excision, VATS, Complications.

\section{INTRODUCTION}

Hydatid disease (HD) is an ancient disease and even was known to Hippocrates. It can virtually affect any organ in the body from head to toe, and most common affected organs are found to be liver and lungs. ${ }^{1}$ Occurrence of hydatid disease at unusual sites is approximately $8-10 \%$. Hydatid disease is still a major health problem in the infested areas of world, mainly in sheep-raising communities. But owing to increased travel and tourism all over the world, it can be found anywhere, even in developed countries. ${ }^{2}$ It can involve any organ and mimic almost any pathological condition. The clinical picture depends upon the involved organs, its effects on adjacent structures, complications due to secondary infection, rupture and anaphylaxis caused by hydatid cysts. ${ }^{3}$ The early asymptomatic phase and delayed presentation makes early diagnosis difficult. The various complications of the disease, rather than the disease itself are more difficult to treat. The disease might undertake a 'malignant' course in the sense of high rate of recurrence, which further enforces the importance of meticulous evaluation and proper planning of treatment and better preventive methods aiming at control and eradication of this age old disease. ${ }^{4}$ Further studies are therefore needed to improve our understanding of the various clinical problems caused by this parasite in general and more specifically to reassess the surgical nature, complications and fatality of this disease in order to apply more appropriate surgical treatment to human Echinococcosis. ${ }^{5}$ Surgery for hydatid disease has always been considered as the gold standard in terms of therapy despite significant advances in medical treatment and interventional radiology. ${ }^{6}$ With the passage of time, the treatment for abdominal hydatid disease is undergoing revolutionary changes and the era of open surgery with its associated large incision and prolonged stay is now being challenged by lesser invasive procedures. ${ }^{7}$ With evolution of laparoscopic surgeries, laparoscopic treatment of hydatid disease has also evolved. ${ }^{8}$ We have undertaken 
this research to study incidence of hydatid disease in various parts of body, its clinical presentation and management with associated complications.

Aims and objectives of the study were to study epidemiology, clinical features and complications at presentation in hydatid cyst in different organs, to study distribution of hydatid cyst in different organs, various modalities of management of hydatid cyst advantages and disadvantages and to study morbidity and mortality in various modalities for management of hydatid cyst.

\section{MATERIAL AND METHODS}

This study was carried out in Department of General Surgery of a medical college situated in an urban area. The study period spanned over 7 years (2010-2017). A total of 148 cases of hydatid disease were included in this study on the basis of a predefined inclusion and exclusion criteria. Detailed history of the patients along with demographic details was noted down. A complete general and systemic examination was done. Diagnosis was confirmed with the help of various investigations like $\mathrm{X}$-ray, ultrasonography and contrast enhanced CT. MRI was done in patients with head and neck hydatid or spinal and musculoskeletal hydatids. Routine investigations like blood group, complete blood count, liver function tests, kidney function tests, blood sugar level, urine analysis and coagulation profile was done in all the cases. Lung function tests were done in patients with thoracic hydatid. 2D echo along with coronary angiography was done in cases of cardiac hydatid. Patients were started on medical management with tablet Albendazole $10 \mathrm{mg} /$ $\mathrm{kg}$ body weight/day (400mg twice a day) preoperatively for at least 2 weeks. After complete evaluation, surgical management was undertaken. Postoperatively albendazole was continued for 3-6 months.

\section{Inclusion Criteria}

1. All the patients admitted in department of surgery with the diagnosis of Hydatid disease.

2. Patient giving informed consent to be part of the study.

3. Patient who followed up at least for 1 year.

\section{Exclusion Criteria}

1. Patient having hydatid disease who have already undergone surgery previously.

2. Recurrent hydatid disease.

3. Patients who refused consent to be part of the study.

4. Patient who didn't come for follow up at least for 1 year.

\section{RESULTS}

Out of total 148 patients there were 46 males and 102 females with a $\mathrm{M}: \mathrm{F}$ ratio of $1: 2.21$. The highest incidence was found in 4th decade (26.35\%) (Figure-1).

The Analysis of age groups of the affected cases showed that the most common age group affected was between 31- 40 years $(26.35 \%)$ followed by $41-50$ years $(18.24 \%)$ and $51-60$ years (16.21\%) (table-1).

Among the 148 patients with hydatid cysts, most of them $67.56 \%$ patients found to have hydatid cysts in liver. Followed by lung, where, $20.27 \%$ patients had hydatid cysts. In these, $4.05 \%$ patients had liver hydatid along with lung hydatid

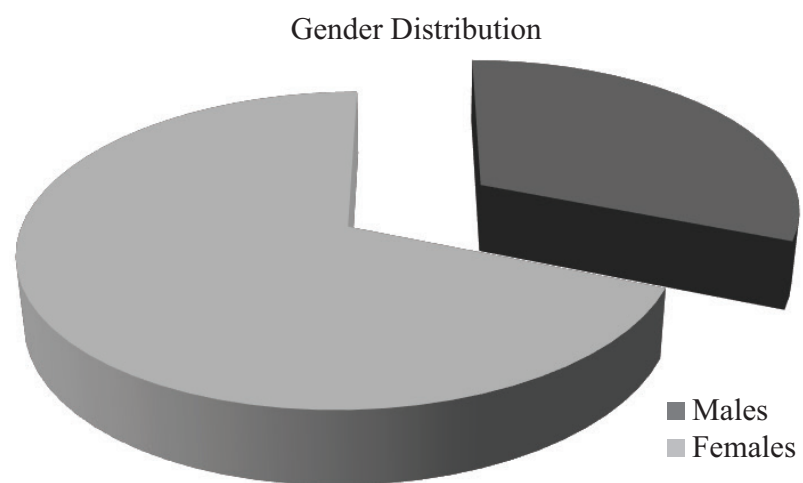

Figure-1: Gender Distribution Of Studied Cases.

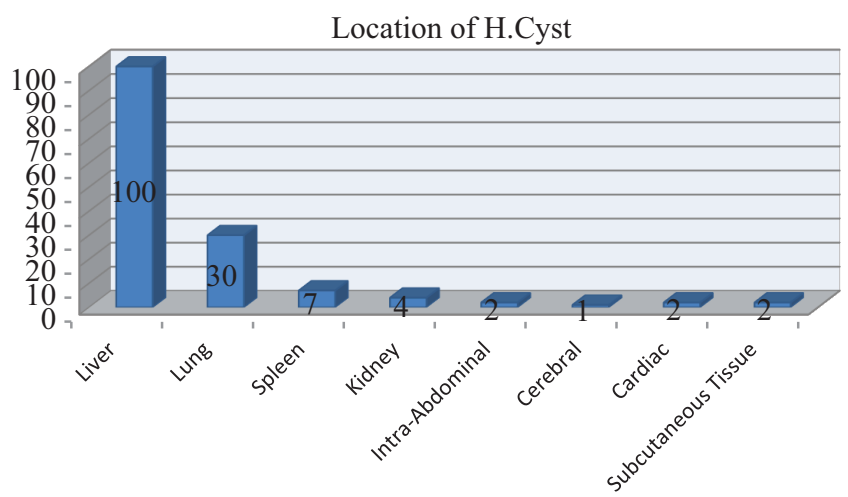

Figure-2: Location Of the Hydatid Cysts

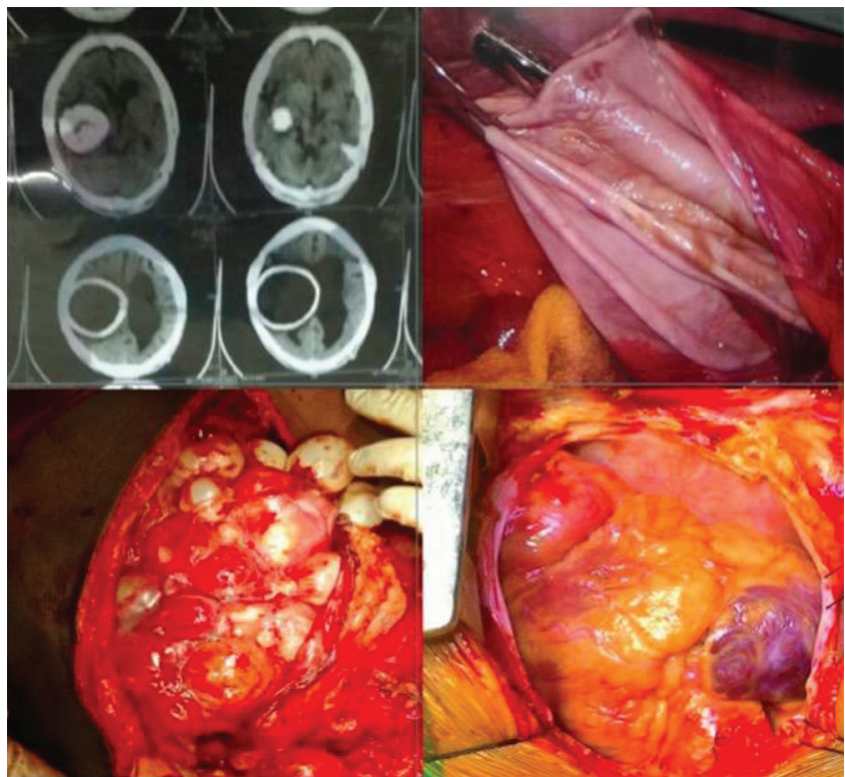

Figure-3: Typical Ring Enhancing Lesion and hydrocephalus seen on Computerized Tomography, Hepatic, Peritoneal and cardiac hydatid cysts (Clockwise from left upper corner).

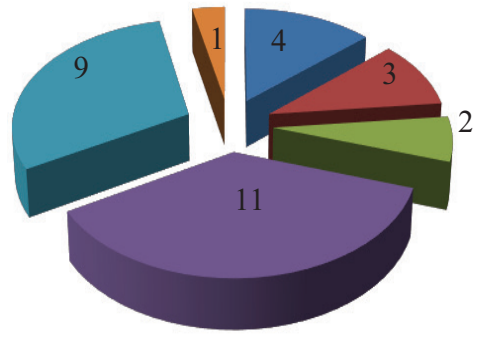

Intrabiliary rupture

- Intrapleural rupture

- Peritoneal Rupture

- Infected Cyst

- Ruptured Lung Hydatid

- Calcified Renal Hydatid

Figure-4: Complication seen in the studied Cases. 


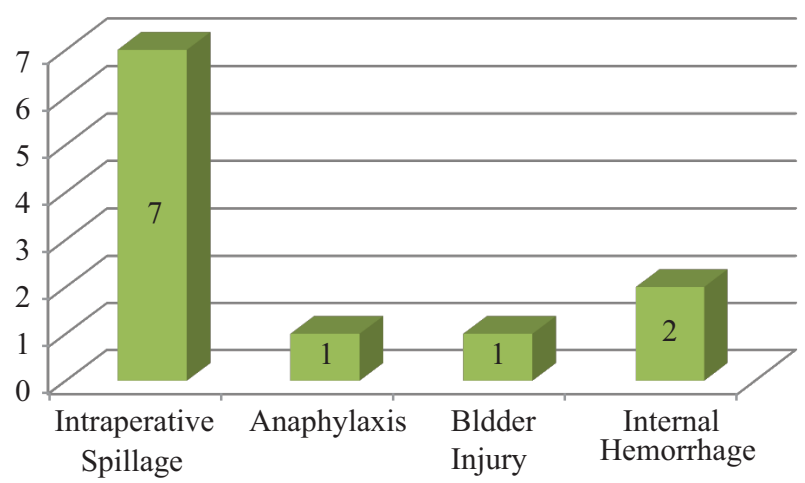

Figure-5: Intraoperative complications in the studied cases.

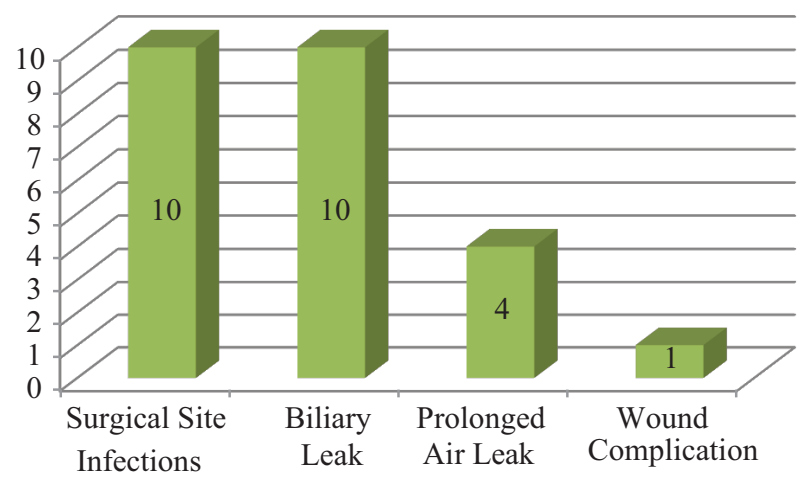

Figure-6: Intra-operative complications in the studied cases.

\begin{tabular}{|l|c|c|}
\hline Age Group & Incidence & Percentage \\
\hline$<10$ yrs & 2 & $1.35 \%$ \\
\hline $10-20$ yrs & 14 & $9.45 \%$ \\
\hline $21-30$ yrs & 26 & $17.56 \%$ \\
\hline $31-40$ yrs & 39 & $26.35 \%$ \\
\hline $41-50$ yrs & 27 & $18.24 \%$ \\
\hline $51-60$ yrs & 24 & $16.21 \%$ \\
\hline$>60$ yrs & 16 & $10.81 \%$ \\
\hline Total & 148 & $100 \%$ \\
\hline \multicolumn{2}{|c|}{ Table-1: Age Distribution of the studied cases. } \\
\hline
\end{tabular}

\begin{tabular}{|l|c|c|c|}
\hline Procedure & Open & $\begin{array}{c}\text { Lap/ } \\
\text { Endoscopic }\end{array}$ & Total \\
\hline Liver & 30 & 70 & 100 \\
\hline Lung & 21 & 9 & 30 \\
\hline Kidney & 3 & 1 & 04 \\
\hline Spleen & 3 & 4 & 07 \\
\hline Subcutaneous & 2 & 0 & 02 \\
\hline Intra-abdominal & 2 & 0 & 02 \\
\hline Cardiac & 2 & 0 & 02 \\
\hline Cerebral & 1 & 0 & 01 \\
\hline Total & 64 & 84 & 148 \\
\hline
\end{tabular}

Table-2: Distribution of the cases on the basis of surgery (Open/Endoscopic)

cysts, and $1.35 \%$ patients had liver hydatid along with splenic hydatid.(see table II) In liver, hydatid was predominated in right lobe with $74 \%$ patients had right liver lobe hydatid, and $13 \%$ had hydatid cyst in left lobe and 13\% had it in both lobes of liver. In lung, right lung found to be most commonly involved with $62.5 \%$ patients with lung hydatid had the cyst in right lung, and $25 \%$ of patients had it in left lung and $12.5 \%$ patients with lung hydatid found to have both lungs affected (figure-2).

In present study most of the patients with liver hydatid presented with abdominal pain (70\%) which predominantly was in right hypochondrium, $15 \%$ patients presented with lump in abdomen, $11 \%$ patients presented with fever. A significant no of patients (33\%) patients were asymptomatic at presentation. In patients presented with lung hydatidosis common signs and symptoms included cough (69.23\%), chest pain (23.33\%), breathlessness (6.66\%), hemoptysis (16.66\%) and fever (6.66\%). All the splenic hydatid patients were presented with lump and pain in abdomen, where 2 patients with renal hydatid were presented with lump and hematuria, and one presented with lump in lumbar region. Patients with cardiac hydatid were asymptomatic, and were diagnosed on routine screening for the cardiovascular diseases, and cerebral hydatid patient presented with seizures. In this study, we found that, $4 \%$ patients of the liver hydatid patients had intrabiliary rupture at presentation, $3 \%$ patients had liver hydatid ruptured into pleural cavity, $2 \%$ patient had silent peritoneal rupture with secondary peritoneal hydatidosis, and $11 \%$ patients had infected hydatid cysts. 9 (30\%) patients with lung hydatid had ruptured hydatid at presentation, and all of them had cyst ruptured into pleural cavity. One renal hydatid patient presented with calcified hydatid cyst (figure-3,4).

All 148 patients included in this study were treated surgically either by open (44.60\%) or endoscopic $(55.40 \%)$ techniques. Among 100 operated liver hydatid cysts, 70\% of the procedures were done with laparoscopic techniques, operated with cystotomy and evacuation of membranes with omentopexy and tube drainage. $30 \%$ patients were operated by open method. In 11 patients from open group initially laparoscopic surgery was attempted but later converted to open procedure. Common reasons for conversion were difficult approach and incomplete evacuation. In a case of pelvic hydatid cyst with liver hydatid during the handling of pelvic cyst iatrogenic organ injury (bladder injury) occurred which was the reason for conversion in one case. Internal bleeding due to liver laceration was the reason for conversion in one case. Adhesions and intra-operative intraperitoneal rupture of the cyst was the reason for conversion in one case each. Among lung hydatid cysts 9 (30\%) were operated with thoracoscopic techniques and 21 (70\%) were operated with open technique. $6(20 \%)$ were initially started with thoracospic technique but later were converted to thoracotomy due to presence of dense adhesions. 2 patients with liver hydatid with rupture into pleural cavity were operated with thoracoabdominal approach. 2 patients had undergone lobectomy and 1 patient had undergone pneumonectomy, due to extensive involvement of cyst and rests of the patients were operated with cyst excision. In hydatid spleen, Splenectomy was done in 6 (85.7\%), as the cyst involved entire spleen with almost no splenic tissue, one patient (14.3\%) cystectomy was done (table-2). In renal hydatid, open nephrectomy has been done. As the cyst was involving entire kidney and causing the parenchymal distortion. In cardiac hydatid, the hydatid was there in 
epicardial location, where excision of the cyst wall was done. 1 more patient with cardiac hydatid had hydatid cyst in pericardium which was excised by thoracoscopic technique. In cerebral hydatid, hydatid was in temporoparital lobe, where the thin cortex was incised and the cyst was removed by Dowling's method.

The Analysis of intra-operative complications showed that Spillage was most dreaded complication in the surgery of the hydatid cyst. Intra operative complications depend on the type of surgical procedure used. We took adequate precautions to avoid spillage of the contents. Although spillage is considered a nightmare during hydatid cyst surgery, its relevance in practice is questionable. In present series, among 100 operated liver hydatid cysts, 7 cases had intra operative spillage and one patient had anaphylaxis following spillage. It was managed adequately with intravenous hydrocortisone and with inotropic support by the anesthetists in the theater. Patient recovered well from the episode post operatively.

Iatrogenic organ injury (bladder injury) was seen in 1 patient while attempting to excise broad ligament hydatid cyst. Internal bleeding was seen in 2 cases which occurred during evacuation of liver hydatid cyst. Rests of the surgeries were uneventful. All the above complications were observed in the laparoscopic surgeries (figure-5).

The analysis Of Postoperative complications showed that out of 100 operated liver hydatid cysts 10 patients developed surgical site infection, which were managed by appropriate antibiotics and dressings. 10 patients developed biliary leak. Out of these 10 patients who developed biliary leak in 7 patients leak subsided within 10 days while in 3 patients biliary drainage persisted for more than 3 weeks and these cases were managed by Endoscopic sphincterotomy. Among 30 operated lung hydatid cyst patients 4 people developed prolonged air leak and later required lobectomy. One splenic hydatid patient developed wound complication and was managed conservatively. Post-operative period of renal, pelvic, cardiac and cerebral hydatid were uneventful (figure-6).

Patients were followed up at 6th month, 1 year and even after that if feasible. We could not find any recurrences till the end of the study period.

\section{DISCUSSION}

Hydatid disease is a zoonotic disease, incidence of which is decreasing now a days, with modified lifestyle of the people and improved sanitation. ${ }^{9}$ We studied 148 patients who were surgically managed for hydatid cysts. In this study we could observed that, most patients had hydatid cysts in liver followed by lungs. Liver acts as the first filter for the cysts, where they lodge and grow and followed by lung which acts as the second filter. We could also find hydatid cysts in liver along with hydatid cysts of other organs like lungs, spleen etc. Cysts grow slowly hence patients remain asymptomatic till it exerts pressure effects on the adjacent tissue with growth of cyst. Thus a patient of hydatid cyst in liver will be asymptomatic, and may later present with dragging pain in right hypochondrium and lump. ${ }^{10}$ In some patients it may present with ruptured cyst into the biliary tract thereby causing obstructive jaundice, or may secondarily get infected, or rupture into the peritoneal cavity. ${ }^{11}$ USG abdomen is a useful investigation in liver hydatids, which aids in determining the size of the cyst, presence of daughter cysts, number of cysts, lobe and segments involved and staging of the cysts. ${ }^{12}$ It also helps to find associated hydatid cysts in other abdominal organ. Liver hydatids can be managed by surgery by either open or laparoscopic techniques, in a conservative approach with cystotomy and evacuation of the membranes and sterilization of the cyst with $2 \%$ cetrimide solution with adequate precaution in cysts with intrabiliary communication. Liver resection or pericystectomy was not done in any of the patients, and we did not find any recurrence in any of our patients under follow up. The fear of the laparoscopic techniques was the spillage of the cyst contents resulting in extensive peritoneal hydatid cysts. ${ }^{13}$

With proper precautions during procedure we could minimize the spillage and with antihelmenthics in postoperative period there were no recurrences in follow up with laparoscopic method. Conversion rate was low as well as post-operative complications were also minimal. However ten patients had biliary drainage in the drain which were kept in the cavity, seven of them were managed conservatively, while three of them had persistent biliary drainage for more than three weeks who were managed with ERCP and sphincterotomy. ${ }^{14}$ Lung was the next most common organ affected by Echinococcosis following the liver, as it is the second filter for the cysts. Lung hydatid cyst patients presented with cough, as the cyst compress the adjacent terminal bronchioles. ${ }^{15}$ When the cyst grows and as the intracystic pressure increases, cyst may get ruptured. We found significant number of patients with ruptured lung hydatid into pleural cavity presenting as pleural effusion which could be seen on chest $\mathrm{x}$-rays. ${ }^{16}$

Video-Assisted thoracoscopic Surgery (VATS) is becoming increasingly popular for management of lung hydatids. ${ }^{17}$ In our study 30\% patients with lung hydatids were managed successfully with VATS and in $15 \%$ of patients procedure was converted from VATS to thoracotomy. And remaining 55\% patients were managed by thoracotomy. Pulmonary hydatid cysts can be treated either by cystectomy or lobectomy and in cases of extensive involvement pneumonectomy may be necessary. ${ }^{18} \mathrm{We}$ did cystectomy in all the patients except with 2 patients undergoing lobectomy and one patient undergoing pneumonectomy due to the extensive involvement by the cyst. Post operatively $10 \%$ patients developed air leak. Who required lobectomy. With no post op recurrences were observed.

Splenic hydatid cysts are usually managed either by cystectomy or Splenectomy. ${ }^{19}$ For splenic hydatid cysts we did Splenectomy in six out of seven patients, due to extensive involvement of cyst. And cystectomy was done in one patient. We used laparoscopic approach in four patients with nil postoperative complications and no recurrences. Renal hydatid may be seen as simple or complex renal cyst on imaging. ${ }^{20}$ In this study renal hydatid patients were managed by nephrectomy. Nephron sparing procedures could not be done as the cysts involved the entire parenchyma with dysfunction of $\mathrm{t}$ he kidney. Cerebral hydatid patients presented with seizures and were diagnosed on the basis of presence of typical ring enhancing lesions on CT brain. 
These patients were operated by craniotomy and Dowling technique.

Postoperative period was uneventful and no recurrences were documented. Amongst two cardiac hydatid patients, one Cardiac hydatid patient whose cyst was located in epicardial location was operated with midline sternotomy and excision of the cyst. Another patient whose cyst was on pericardium was operated with endoscopic technique. Postoperative period was uneventful in both the patients.

\section{CONCLUSION}

Hydatid cysts can practically involve any organ. Patients may get infested during childhood and may present later in life. Cyst prefers to grow in low pressure zones and tend to grow towards surface and exerts pressure effects on adjacent structures due to which patients may develop symptoms. During its course of enlargement the cyst if untreated can develop complications. Management of the hydatid cyst can safely be done by open surgery, laparoscopic surgery or VATS with least complications. Meticulous surgery along with oral antihelmenthics (Albedazole) reduced the risk of recurrence to negligible level.

\section{REFERENCES}

1. Pump KK. Echinococcosis (Hydatid Disease): Review and Report of a Case of Secondary Echinococcosis. Canadian Medical Association Journal. 1963;89(2):7378.

2. Barnes TS, Deplazes P, Gottstein B, Jenkins DJ, Mathis A, Siles-Lucas M, Torgerson PR, Ziadinov I, Heath DD. Challenges for diagnosis and control of cystic hydatid disease. Acta Trop. 2012;123(1):1-7.

3. Vuitton DA. Echinococcosis and allergy. Clin Rev Allergy Immunol. 2004;26(2):93-104.

4. Irabedra P, Ferreira C, Sayes J, et al. Control programme for cystic echinococcosis in Uruguay. Memórias do Instituto Oswaldo Cruz. 2016;111(6):372-377.

5. Torres RR and Campbell JR. An interpretetive review of the surgical treatment of hydatid disease. Surgery Gynaecology and obstetric 1965;121(2): 851-861.

6. C Palanivelu et al. Laparosocpic management of hepatic hydatid disease. JSLS 2 006;10(1):56 -62.

7. Buia A, Stockhausen F, Hanisch E. Laparoscopic surgery: A qualified systematic review. World Journal of Methodology. 2015;5(4):238-254.

8. Zaharie F, Bartos D, Mocan L, Zaharie R, Iancu C, Tomus C. Open or laparoscopic treatment for hydatid disease of the liver? A 10-year single-institution experience. Surgical Endoscopy. 2013;27(6):2110-2116.

9. Ozer N, Aytemir K, Kuru G, Atalar E, Ozer Y, Ovunc $\mathrm{K}$, et al. Hydatid cyst of the heart as a rare cause of embolization: report of 5 cases and review of published reports. J Am Soc Echocardiogr 2001;14(5):299-302.

10. De U. Primary abdominal hydatid cyst presenting in emergency as appendicular mass: a case report. World Journal of Emergency Surgery : WJES. 2009;4(1):13

11. Turkyilmaz Z, Kapisiz A, Sonmez K, Karabulut R, Basaklar A. Obstructive jaundice resulted from hydatid cyst of pancreatic head. Hippokratia. 2013;17(4):378379 .
12. Turgut AT, Akhan O, Bhatt S, Dogra VS. Sonographic spectrum of hydatid disease. Ultrasound Q. 2008;24(1):17-29.

13. Jani K. Spillage-free laparoscopic management of hepatic hydatid disease using the hydatid trocar canula. Journal of Minimal Access Surgery. 2014;10(3):113118.

14. Al-Shareef Z, Hamour OA, Al-Shlash S, Ahmed I, Mohamed AA. Laparoscopic Treatment of Hepatic Hydatid Cysts With a Liposuction Device. JSLS : Journal of the Society of Laparoendoscopic Surgeons. 2002;6(4):327-330.

15. Sarkar M, Pathania R, Jhobta A, Thakur BR, Chopra R. Cystic pulmonary hydatidosis. Lung India : Official Organ of Indian Chest Society. 2016;33(2):179-191.

16. Garg MK, Sharma M, Gulati A, et al. Imaging in pulmonary hydatid cysts. World Journal of Radiology. 2016;8(6):581-587.

17. Uchikov AP, Shipkov CD, Prisadov G. Treatment of lung hydatidosis by VATS: a preliminary report. Canadian Journal of Surgery. 2004;47(5):380-381.

18. Salih OK, Topcuoğlu MŞ, Çelik ŞK, Ulus T, Tokcan A. Surgical treatment of hydatid cysts of the lung: analysis of 405 patients. Canadian Journal of Surgery. 1998;41(2):131-135.

19. Meimarakis G, Grigolia G, Loehe F, Jauch K, Schauer R. Surgical management of splenic echinococcal disease. European Journal of Medical Research. 2009;14(4):165170.

20. Bhaya A, Shinde AP. Isolated renal hydatid presenting as a complex renal lesion followed by spontaneous hydatiduria.World Journal of Radiology. 2015;7(7):180183.

\section{Source of Support: Nil; Conflict of Interest: None}

Submitted: 09-04-2018; Accepted: 04-05-2018; Published online: 16-05-2018 